drawback that it needs to be repeated every time a different input state is chosen. Konrad et al. ${ }^{2}$ look at the problem from a fresh perspective (Fig. 1c). Instead of calculating the time evolution for each input state separately, they consider the dynamics of one particular state - the so-called maximally entangled state - and derive from this analysis general statements valid for arbitrary input states. As an important tool, they used a one-to-one mapping between quantum channels and quantum states that has been known for more than thirty years ${ }^{6}$, and has many applications in quantum information theory. Konrad and colleagues ${ }^{2}$ use this isomorphism to exchange the role of the quantum state and the quantum channel and demonstrate that in this 'dual picture', the original problem can be solved more efficiently. For pure input states, they arrive at a factorization law stating that the entanglement of the final state, $\rho_{\mathrm{f}}$, can be expressed as the product of the initial entanglement, $C\left(\rho_{\mathrm{i}}\right)$, multiplied by the entanglement reduction induced by the passage through the quantum channel; the latter turns out to be universal for all quantum states entering the channel. These findings can be generalized to mixed input states and to concatenation of quantum channels, at the price, however, of turning the factorization law into an inequality. Most importantly, the new technique provides a direct way of inferring the dynamical evolution of entanglement under the action of a decohering environment.

The work by Konrad et al. ${ }^{2}$ elegantly deals with the case of two entangled qubits. But further ideas will be necessary to extend the results to general composite quantum systems as the derivation relies heavily on the availability of an entanglement measure; for two qubits, such a measure is easy to calculate, but no equivalent is available for higher-dimensional systems. This notwithstanding, the results of Konrad and colleagues add an important piece to our understanding of entanglement and its dynamical aspects.

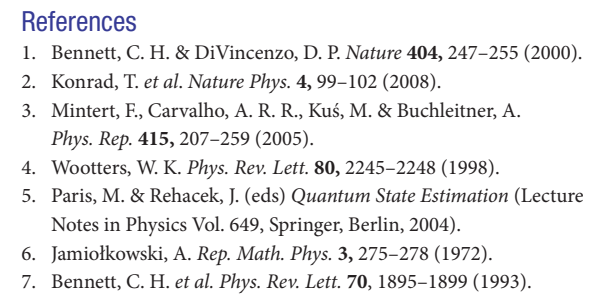

References

$247-255(2000)$

ys. Rep. 415, 207-259 (2005).

7. Bennett, C. H. et al. Phys. Rev. Lett. 70, 1895-1899 (1993)

\title{
ASTROPHYSICS
}

\section{Rings around the lenses}

During a routine Hubble Space Telescope scan for an Einstein ring - a galaxy with a bright ring around it - astronomers noticed a double ring, the first of its kind. The team led by Raphael Gavazzi and Tommaso Treu report their discovery online (http://arxiv.org/abs/0801.1555; 2008).

A single Einstein ring is a consequence of gravitational lensing, whereby a massive galaxy (the lens) bends the light from a more distant galaxy (the object) along the same line of sight, or optical axis. Rather than focusing the light, as lenses do, the galaxy in the foreground has a focal line that creates mirages of the object, so we see the deflected light as a ring around the lens instead of a spot. (When the alignment is not perfect, arcs will appear instead of a full ring.) Acting as a magnifier, the lens amplifies the brightness of the object.

Albert Einstein himself made the calculation for lensing by a single star, which didn't yield an easily observable ring, but Fritz Zwicky was the first to propose that galaxies can also act as gravitational lenses. In fact, any massive object can bend space-time.

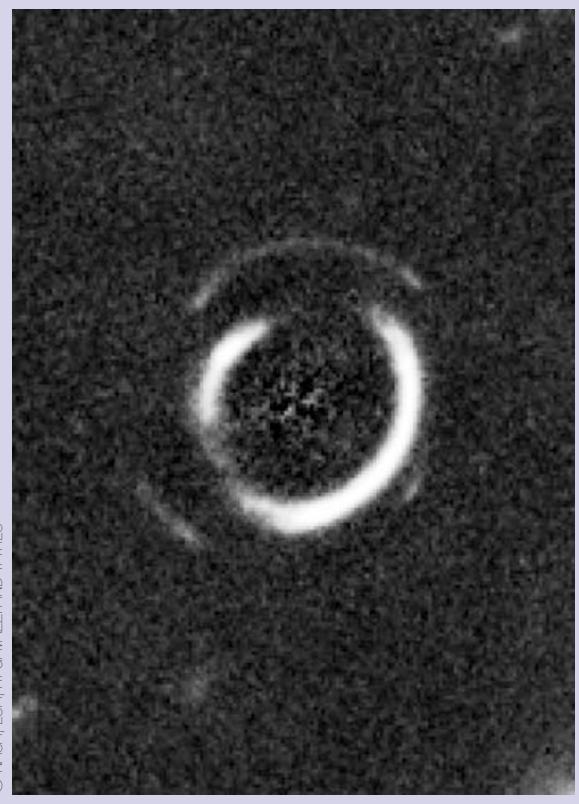

double ring, which is produced by light from not one but two distant galaxies beyond the one we can see, the situation is even better. The galaxy in the middle acts as an additional lens for the most distant one (to create the outer ring). Of course, the compound lens is more difficult to model, but it provides a unique method of measuring the total mass of small distant galaxies.

At 3 billion, 6 billion and approximately 11 billion light-years away, the galaxies in the double Einstein ring are cosmologically distant. A natural question then is whether we can obtain constraints on cosmological parameters. According to Gavazzi et al., the uncertainties are currently too large for any meaningful interpretations. However, they calculate that fifty such double-source lens systems would lead to measurements of the matter density of the Universe and the equation of state of dark energy with unprecedented accuracy (10\%). With several planned space missions expected to reveal tens of thousands of single Einstein rings and tens of double Einstein rings, that goal may not be so far away.

May Chiao 